human-derived severe fever with thrombocytopenia syndrome virus isolates from South Korea. PLoS Negl Trop Dis. 2017;11:e0005893. http://dx.doi.org/10.1371/ journal.pntd.0005893

7. Kumar S, Stecher G, Tamura K. MEGA7: Molecular evolutionary genetics analysis version 7.0 for bigger datasets. Mol Biol Evol. 2016;33:1870-4. http://dx.doi.org/10.1093/ molbev/msw054

8. Lin TL, Ou SC, Maeda K, Shimoda H, Chan JPW, Tu WC, et al. The first discovery of severe fever with thrombocytopenia syndrome virus in Taiwan. Emerg Microbes Infect. 2020;9:14851. http://dx.doi.org/10.1080/22221751.2019.1710436

9. Tsai YL, Shyu CL, Yao CT, Lin JA. The ixodid ticks collected from dogs and other animals in Taiwan and Kinmen Island. Int J Acarol. 2012;38:110-5. http:/ / dx.doi.org/10.1080/ 01647954.2011.594812

10. Chao LL, Lu CW, Lin YF, Shih CM. Molecular and morphological identification of a human biting tick, Amblyomma testudinarium (Acari: Ixodidae), in Taiwan. Exp Appl Acarol. 2017;71:401-14. http://dx.doi.org/10.1007/ s10493-017-0119-9

Address for correspondence: Pei-Yun Shu, Vector-Borne Viral and Rickettsial Diseases Laboratory, Center for Diagnostics and Vaccine Development, Centers for Disease Control, Ministry of Health and Welfare, Taipei 11561, Taiwan; email: pyshu@cdc.gov.tw

\section{Lesions of Mycobacterium avium spp. hominissuis Infection Resembling M. bovis Lesions in a Wild Mule Deer, Canada ${ }^{1}$}

\author{
Kirsten M.F. Frayne, Brock R. Chappell, \\ Jennifer L. Davies, Bryan J. Macbeth, \\ Musangu Ngeleka, Jamie L. Rothenburger
}

Author affiliations: University of Calgary Faculty of Veterinary Medicine, Calgary, Alberta, Canada (K.M.F. Frayne, B.R. Chappell, J.L. Davies, J.L. Rothenburger); Banff National Park, Parks Canada Agency, Banff, Alberta, Canada (B.J. Macbeth); Prairie Diagnostic Services, Saskatoon, Saskatchewan, Canada (M. Ngeleka); Canadian Wildlife Health Cooperative Alberta Region, Calgary (J.L. Rothenburger)

${ }^{1}$ Preliminary results from this study were presented at the American College of Veterinary Pathologists Annual Meeting, November 9-13, 2019, San Antonio, Texas, USA
We used molecular analyses to confirm Mycobacterium avium spp. hominissuis infection in lung granulomas and pyogranulomas in the tracheobronchial lymph node in a wild mule deer in Banff, Canada. These lesions are similar to those found in M. bovis-infected animals, emphasizing the critical need for disease surveillance in wildlife populations.

DOI: https://doi.org/10.3201/eid2607.200187

Tn November 2018, a wild yearling male mule Ideer (Odocoileus hemionus) was found dead in Banff National Park, Alberta, Canada. The carcass was submitted to the Canadian Wildlife Health Cooperative Alberta Region at the University of Calgary (Calgary, Alberta, Canada) for diagnostic investigation. The University of Calgary Veterinary Sciences Animal Care Committee approved this research (AC17-0010).

Necropsy revealed that the deer had died of blunt-force trauma, presumably having been struck by a vehicle. During the necropsy, we found mineralized granulomas in the right caudal lung lobe and multifocal pyogranulomatous lymphadenitis in the tracheobronchial lymph node (Figure). We fixed lesion samples in 10\% neutral buffered formalin for 48 $\mathrm{h}$, then processed the samples by routine methods; we stained 4- $\mu m$-thick sections of paraffin-embedded tissues with hematoxylin and eosin before examination with light microscopy by an anatomic veterinary pathologist (J.L.R.), certified by the American College of Veterinary Pathologists.

Histopathology of the lung confirmed granulomatous pneumonia. Affected multifocal areas were characterized by central necrotic debris that was variably mineralized, surrounded by macrophage aggregates, multinucleated giant cells, lymphocytes, plasma cells, and variably thick fibrous capsules. Histopathology of the lymph node revealed similar multifocal areas of necrosis, variable mineralization, and infiltration of macrophages in the edges of some affected areas. Because of autolysis and freeze-thaw artifact, further characterization of the inflammatory cell population was not possible.

We designated the lymph node lesions as pyogranulomatous lymphadenitis because of the suppurative gross appearance of the lymph node combined with the microscopic presence of macrophages and mineralization revealed during histologic examination. We identified acid-fast organisms in multifocal macrophages at the margin of necrotic areas in both the lung and lymph node lesions. We submitted frozen samples to the Prairie Diagnostic Services laboratory 

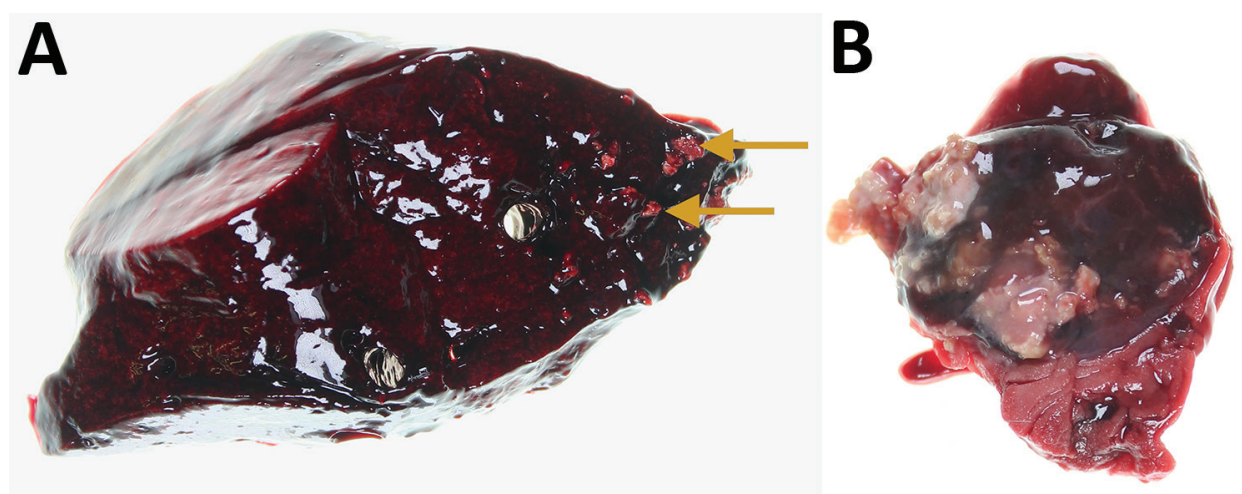

Figure. Lung and tracheobronchial lymph nodes from a wild mule deer (Odocoileus hemionus) infected with Mycobacterium avium spp. hominissuis, Banff National Park, Alberta, Canada. A) Mineralized granulomas in the right caudal lung lobe (arrows). B) Pyogranulomatous lymphadenitis of a tracheobronchial lymph node. The lesions resemble those caused by infection with $M$. bovis.

(Saskatoon, Saskatchewan, Canada), for further analyses. Results of routine bacterial culture and PCR for Mycobacterium bovis were both negative. Subsequently, results were positive from a generic Mycobacterium spp. PCR of the lung and lymph node using nested internal primers of $439 \mathrm{bp}$, as described elsewhere (1). Genomic sequencing analysis identified M. avium spp. hominissuis (GenBank accession no. MT012364).

M. avium complex disease, caused by 4 species of M. avium, including M. avium spp. hominissuis, is considered a potentially zoonotic disease of global importance (4). M. avium spp. hominissuis is a rare nontuberculosis mycobacterium that infects a wide range of species, most commonly humans and pigs $(2,3)$. An opportunistic environmental pathogen that persists in soil and water, M. avium spp. hominissuis typically infects the lungs and intestinal tract, presumably by inhalation and ingestion, respectively $(4,5)$.

In humans, M. avium spp. hominissuis typically results in chronic granulomatous infections in the lungs and cervical lymph nodes (6), most commonly in immunocompromised persons (3). In children, infection causes lymphadenitis of the head and neck region (3). In pigs and other animals, however, $M$. avium spp. hominissuis infection primarily manifests as gastrointestinal disease, with granulomatous lesions in mesenteric lymph nodes and abdominal organs, such as the liver, spleen, and small intestines (6). Yoshida et al. described lung granulomas as an incidental finding in a slaughtered steer that were similar to the ones identified in the mule deer in this study (7). However, these lesions are an unusual manifestation for this bacterium in animals. More typically, respiratory $M y$ cobacterium infections in cattle are caused by M. bovis, the causative agent of bovine tuberculosis and a zoonotic pathogen of global importance with extensive regulatory and trade implications (8).

The lesions in this case were morphologically similar to those described in animals infected with
M. bovis. Pyogranulomas and granulomas in the lungs and cervical lymph nodes are found in wildlife reservoirs of M. bovis, including cervids (8). The similarity of these lesions emphasizes the importance of continuing surveillance and thorough investigation of suspected $M$. bovis cases. Cattle in Canada are regarded as free of $M$. bovis; however, 3 separate cattle herds in western Canada have tested positive from 2011 through March 2020 (9).

M. bovis is endemic in wild wood bison (Bison bison athabascae) and elk (Cervus canadensis) in 2 other national parks in Canada geographically separated from Banff by hundreds of kilometers (10). The presence of wildlife reservoirs elsewhere, combined with the sporadic identification of $M$. bovis-infected cattle herds in Canada, has led to concerns over surveillance in wildlife $(8,9)$. This case demonstrates that routine disease surveillance activities in wildlife populations, including molecular investigations, are crucial to providing ongoing assurance to agricultural and public health sectors of the absence of $M$. bovis in wild cervid populations outside of known endemic areas.

\section{Acknowledgments}

We thanks Susan Calder-Lodge, Jennifer Larios, Melencio Nicolas, Chris Bergeron, Jim Carlsen, and Betty Pollock for invaluable technical support.

Funding and logistical support for this case was provided by the Faculty of Veterinary Medicine, University of Calgary.

\section{About the Author}

Ms. Frayne is a final-year veterinary medicine student at the University of Calgary. She is interested in ecosystem and public health and has a background in both wildlife and laboratory animal medicine. 


\section{References}

1. Telenti A, Marchesi F, Balz M, Bally F, Böttger EC, Bodmer T. Rapid identification of mycobacteria to the species level by polymerase chain reaction and restriction enzyme analysis. J Clin Microbiol. 1993;31:175-8. https:/ / doi.org/10.1128/ JCM.31.2.175-178.1993

2. Klotz D, Barth SA, Baumgärtner W, HewickerTrautwein M. Mycobacterium avium subsp. hominissuis infection in a domestic rabbit, Germany. Emerg Infect Dis. 2018;24:596-8. https:/ / doi.org/10.3201/eid2403.171692

3. Agdestein A, Olsen I, Jørgensen A, Djønne B, Johansen TB Novel insights into transmission routes of Mycobacterium avium in pigs and possible implications for human health. Vet Res (Faisalabad). 2014;45:46. https:/ / doi.org/ 10.1186/1297-9716-45-46

4. Ignatov D, Kondratieva E, Azhikina T, Apt A. Mycobacterium avium-triggered diseases: pathogenomics. Cell Microbiol. 2012;14:808-18. https://doi.org/10.1111/j.1462-5822.2012. 01776.x

5. Nishiuchi $Y$, Iwamoto T, Maruyama F. Infection sources of a common non-tuberculous mycobacterial pathogen, Mycobacterium avium complex. Front Med (Lausanne). 2017;4:27. https://doi.org/10.3389/fmed.2017.00027

6. Komatsu T, Inaba N, Kondo K, Nagata R, Kawaji S, Shibahara T. Systemic mycobacteriosis caused by 'Mycobacterium avium subspecies hominissuis' in a 14-monthold Japanese black beef steer. J Vet Med Sci. 2017;79:1384-8. https://doi.org/10.1292/jvms.17-0204

7. Yoshida S, Araki T, Asai T, Tsuyuguchi K, Arikawa K, Iwamoto T, et al. Phylogenetic uniqueness of Mycobacterium avium subspecies hominissuis isolated from an abnormal pulmonary bovine case. Infect Genet Evol. 2018;62:122-9. https://doi.org/10.1016/j.meegid.2018.04.013

8. Fitzgerald SD, Kaneene JB. Wildlife reservoirs of bovine tuberculosis worldwide: hosts, pathology, surveillance, and control. Vet Pathol. 2013;50:488-99. https://doi.org/ $10.1177 / 0300985812467472$

9. Canada Food Inspection Agency, Government of Canada. Bovine tuberculosis. 2019 [cited 2020 Jan 13] https:/ / inspection.gc.ca/animal-health/terrestrialanimals/diseases/reportable/bovine-tuberculosis/ eng/1330205978967/1330206128556

10. Wobeser G. Bovine tuberculosis in Canadian wildlife: an updated history. Can Vet J. 2009;50:1169-76.

Address for correspondence: Jamie L. Rothenburger, Faculty of Veterinary Medicine, University of Calgary, 3280 Hospital Dr NW, Calgary, Alberta T2N 4Z6, Canada; email: jamie. rothenburger@ucalgary.ca.

\section{Public Mental Health Crisis during COVID-19 Pandemic, China}

\author{
Lu Dong, Jennifer Bouey \\ Author affiliations: RAND Corporation, Santa Monica, California, \\ USA (L. Dong); RAND Corporation, Arlington, Virginia, USA \\ (J. Bouey); Georgetown University, Washington, DC, USA (J. Bouey)
}

DOI: https://doi.org/10.3201/eid2607.200407

The 2019 novel coronavirus disease emerged in China in late 2019-early 2020 and spread rapidly. China has been implementing emergency psychological crisis interventions to reduce the negative psychosocial impact on public mental health, but challenges exist. Public mental health interventions should be formally integrated into public health preparedness and emergency response plans.

China was the first country affected by the pandemic of 2019 novel coronavirus disease (COVID-19), caused by severe acute respiratory syndrome coronavirus 2. Several unique characteristics of China's COVID-19 epidemic patterns and its management policy prompted a heightened public mental health crisis. First, many Chinese residents still remember the 2003 outbreak of severe acute respiratory syndrome (SARS) and its effect on China's social life and economy (1). COVID-19 is more transmissible than SARS, and the case-fatality rate $(2.3 \%)$ is substantially higher than that for seasonal influenza (2). The uncertain incubation period of the virus and its possible asymptomatic transmission cause additional fear and anxiety. Second, the government's initial downplaying of the epidemic's severity eroded public trust in the government's decision-making transparency and competency. Third, unprecedented large-scale quarantine measures in all major cities, which essentially confine residents to their homes, are likely to have a negative psychosocial effect on residents (3). Fourth, reports of shortages of medical protective supplies, medical staff, and hospital beds in Wuhan and the surrounding areas soon followed the citywide quarantine and caused enormous concern throughout the nation. Last, a unique "infodemic" an overabundance of (mis)information on social media (4) and elsewhere-poses a major risk to public mental health during this health crisis.

As during the 2003 SARS and 2014 Ebola virus disease outbreaks, generalized fear and fear-induced overreactive behavior were common among the public; both can impede infection control $(5,6)$. In addition, psychiatric disorders, such as depression, 